\title{
Prevalence of Ureaplasma urealyticum, Mycoplasma hominis and Chlamydia trachomatis in patients with uncomplicated recurrent urinary tract infections
}

\author{
Jadranka Vlasic-Matas ${ }^{1 *}$, Hrvoje Raos ${ }^{2}$, Marijana Vuckovic ${ }^{2}$, Stjepan Radic ${ }^{2}$ and Vesna Capkun ${ }^{3}$ \\ ${ }^{1}$ Polyclinic Nephrology Department, Split, Croatia \\ ${ }^{2}$ School of Medicine, University of Split, Split, Croatia \\ ${ }^{3}$ Department of Nuclear Medicine, Split University Hospital Center, Split, Croatia
}

\begin{abstract}
Aim: To assess the prevalence of Ureaplasma urealyticum, Mycoplasma hominis and Chlamydia trachomatis in patients with chronic urinary tract infections (UTIs) and its correlation with leukocyturia and symptoms.

Methods: The study included 220 patients (130 women and 90 men) presenting with chronic voiding symptoms and sterile leukocyturia. Urine, urethral swabs and cervical swabs (for women patients) were taken to determine the presence of these pathogens. Patients were treated by tetracycline and followed up three and six months after initial therapy.

Results: In 186 (85\%) out of 220 patients, U. urealyticum was found, while C. trachomatis was present in 34 patients (15\%). In majority of female patients (112 out of $130 ; 86 \%)$ U. urealyticum was found. In addition to ureaplasma, in eight patients $M$. hominis was found. C. trachomatis was identified in 18 female patients (14\%). In 74 out of $90(82 \%)$ male patients $U$. urealyticum was detected while in six of them $M$. hominis was also found. C. trachomatis was identified in 16 male patients (18\%). U. urealyticum was significantly related to leukocyturia, as opposed to C. trachomatis $(\mathrm{p}<0,001)$. Women had more frequent symptomatology $(\mathrm{p}=0,015)$ and higer leukocyturia $(\mathrm{p}<0.001)$.
\end{abstract}

Conclusion: Leukocyturia is more common find in U. Urealyticum infection than in patients with C. trachomatis infection. It is therefore advisable to test patients with uncomplicated reccurent UTIs for the presence of these atypical pathogens. Symptomatic patients, as well as their partners, should be treated accordingly.

\section{Introduction}

Mycoplasma hominis (M. hominis) and Ureaplasma urealyticum (U. urealyticum) and Ureaplasma parvum are species in the family Mycoplasmataceae, encompassing the smallest bacteria replicating in culture medium. These tiny microorganisms can be found commensal in lower genitourinary tracts of sexually active men and women. Moreover, they cause many disorders such as non-gonococcal urethritis (NGU), postpartum fever, infertility, and pelvic inflammatory disease $[1,2]$. Their occurrence in the context of urogynaecological diseases has been demonstrated not only in urethritis and cystitis but also in upper renal tract infections, while their role in hyperactive bladder and interstitial cystitis/painful bladder syndrome remains controversial [3].

Chlamydia trachomatis is an obligate intracellular bacterium. During its unique developmental cycle, two different forms are observed, elementary bodies (EBs), which are infectious but not able to divide, and reticulate bodies (RBs), which are metabolically active and able to multiply [4] C. trachomatis is the most common bacterium causing sexually transmitted infections. Most of these infections are asymptomatic and, if not treated, can lead to severe complications, mainly in young women [5].

All of the above-mentioned bacteria are associated with various diseases of the urogenital tract, but they are usually not detected by routine microbiological diagnosis (negative urine cultures can be seen). Urine analysis usually helps in diagnosis. Sterile pyuria is classified as the presence of more than 5-8 leukocytes per highpower field on microscopy, in the setting of negative urine cultures [6,7]. According to previous studies urogenital mycoplasmas and C. trachomatis are frequently found in patients with sterile pyuria [8]. The clinical presentations for sexually transmitted infections (STIs) and urinary tract infections (UTIs) may overlap, and symptoms of dysuria and urinary frequency/urgency occur with both STIs and UTIs $[9,10]$. There are scarce literature data regarding the presence of urogenital mycoplasmas and chlamydia in relation to persistent leukocyturia and chronic symptoms. Therefore, we decided to detect the presence of Ureaplasma urealyticum, Mycoplasma hominis and C. trachomatis in patients experiencing chronic urinary symptoms and their correlation with leukocyturia.

${ }^{*}$ Correspondence to: Jadranka Vlasic-Matas, Polyclinic Nephrology Department, Split, Croatia, Tel: 0038521317320; Fax: 0038521317322; E-mail: jadranka.vlasic-matas@st.t-com.hr

Key words: sterile leukocyturia, uncomplicated recurrent UTIs, Ureaplasma urealyticum, Mycoplasma hominis, Chlamydia trachomatis

Received: January 24, 2019; Accepted: January 31, 2019; Published: February 04, 2019 


\section{Materials and methods}

\section{Patients}

The research was carried out from December 2013 to December 2017. In this period 580 patients were diagnosed with UTI. The study included patients (male and female) older than 18 years of age who had at least three uncomplicated UTIs in the previous 12 months (cystitis or urethritis) and who experienced persistent voiding difficulties despite treatment and/or leukocyturia (defined as the presence of more than 5 leukocytes per high power field on microscopy). Patients who reported antibiotic use in the previous month, with febrile condition, bacteriuria, positive urine culture, complicated UTIs, kidney diseases, neurogenic bladder, on immunosuppression, diabetes mellitus and pregnancy were excluded from the study.

In our study, we only tested patients with symptomatic uroinfection while we treated their partners without testing since it is a sexually transmitted agent. We explained in detail to each patient the importance of taking therapy for both partners regardless of the fact that the other partner had no symptoms.

Were there more partners? It is difficult to answer such a question because the population of the examined patients were not just young people, and we did not include such questions in the research. However, in conversation with the patients we would inquire about that issue as well.

Patients who were included in the study underwent following tests: detailed anamnesis, ultrasound of urotract, urine analysis and microbiological tests. Clinical parameters included symptoms: discomfort when urinating; urinary frequency (polaisuria) and nocturia, feeling of incomplete voiding, dysuria and suprapubic pain.

Urine samples, urethral swabs and cervical swabs (in women) were analysed. Microscopic examination, urine culture and detection of Mycoplasma hominis, Ureaplasma urealyticum and Chlamydia trachomatis was done on all samples initially and after three and six months. The first line therapy drug was tetracycline while the second line of therapy for the patients with positive tests at check-up visit was levofloksacin.

\section{Urine analysis and culture}

A midstream specimen of the first morning urine (clean-catch specimen) was collected for urine analysis and culture test. Only patients with sterile urine culture were included.

For the microscopic examination, a fresh sample of the first morning urine (clean-catch, midstream urine) $10 \mathrm{ml}$ was taken, which was centrifuged for $5 \mathrm{~min}$. at $2000 \mathrm{rpm}$. After centrifugation, the supernatant is discarded, and a volume of 0.2 to $0.5 \mathrm{ml}$ is left inside the tube. The sediment is resuspended in the remaining supernatant by manually shaking the tube. A drop of resuspended sediment is poured onto a glass slide and covered with a slip. The urine sediment specimen is first viewed with $10 \mathrm{x}$ magnification and then $40 \mathrm{x}$ magnification in at least 10 visual fields, and the results were expressed as an average number of cells.

It is considered that in the urine of a healthy person there can be up to five leukocytes per visual field. More than five leukocytes per visual field is considered to be leukocyturia. The presence of $\geq 10$ white blood cells/high-power field is indicative of pyuria [11]. As for erythrocytes, normal value was considered the number of up to three $\mathrm{E}$ in visual field.

\section{Detection of urogenital mycoplasmas and C. trachomatis}

The first voided morning specimen was taken in order to prove the presence of $U$. urealyticum, M. hominis and C. trachomatis respectively. In symptomatic patients without leukocyturia, urethral swab and cervical swab in women were also analysed. For urethral swabs, patients were not allowed to urinate at least three hours before sample taking. The presence of urogenital mycoplasmas, i.e, Ureaplasma urealyticum/parvum and Mycoplasma hominis from urogenital specimens was determined using commercially available MycoView kit (Zeakon diagnostics, France). The kit enables identification, quantification and susceptibility testing to five antibiotics: levofloxacin, moxifloxacin, erythromycin, clindamycin and tetracycline. We considered positive finding $>10^{4}$.

Unfortunately, in our regional medical institutions PCR identification of $M$. hominis and U. urealyticum is not performed jet. Chlamydia trachomatis was detected by real time PCR using Cobas 4800 CT/NG test (Roche) after automatic extraction (Cobas x 480, Roche Diagnostics) and amplification (Cobas z 480, Roche Diagnostics).

Data entered in excel table were processed in IBM SPSS Statistics 20. In data processing we used $\chi 2$ test, Mann Whitney $U$ test. Results were interpreted at a significance level $\mathrm{p}<0.05$.

Statistical analysis included data referring only to the first patients visit.

\section{Results}

The research included 220 patients (130 women and 90 men) who met the inclusion criteria. In 186 out of 220 patients (85\%) $U$. urealyticum was found while C. trachomatis was present in 34 patients (15\%). Hundred and twelve out of 130 (86\%) female patients had $U$. urealyticum while in eight patients $M$. hominis was also detected. $C$. trachomatis was found in 18 female patients (14\%). In 74 out of 90 male patients $(82 \%)$ U. urealyticum was found; while in six of them $M$. hominis was also detected. C. trachomatis was detected in 16 male patients (18\%).

Mean age of patients included in the study and the number of leukocytes and erythrocytes in urine is presented in Table 1.

Women patients included in the study were significantly younger than men. Leukocyturia in female patients were significantly higher than in men while the erythrocytes count was significantly lower. Leukocyturia differed significantly regarding the pathogen found. Patients with cultures positive to Ureaplasma urealyticum had significantly higher leukocyte counts than patients with Chlamydia trachomatis detected, as seen in Table 2. Median of leukocytes in urine of patients with positive U. urealyticum was for 11 leukocytes $(95 \%$ CI: 7-15 leukocyte) higher than in patients with C. trachomatis $(\mathrm{z}=4.4$; $\mathrm{r}=0.31 ; \mathrm{p}<0.001)(\mathrm{r}=$ effect size $)$.

We did not prove statistically significant difference in erythrocyte number between patients with U. urealyticum and C. trachomatis. In Table 3. there is a correlation of symptoms in relation to gender.

Distribution of patients according to symptoms in relation to gender was statistically significantly different $(\chi 2=62.7 ; p<0.001)$. Strength of assosiation is moderate (Cramers V $=0.543 ; \mathrm{p}<0.001$ ).

The proportion of only one symptom in the form of discomfort during urinating in men was nine times higher than the proportion of the same problem in women. The proportion of female patients with 
Vlasic-Matas J (2019) Prevalence of Ureaplasma urealyticum, Mycoplasma hominis and Chlamydia trachomatis in patients with uncomplicated recurrent urinary tract infections

Table 1. Presentation of research variables of all patients in relation to gender

\begin{tabular}{|c|c|c|c|c|c|}
\hline Variables & & $\begin{array}{l}\text { All patients } \\
(\mathbf{n}=\mathbf{2 2 0})\end{array}$ & $\begin{array}{c}\text { Men } \\
(n=90)\end{array}$ & $\begin{array}{l}\text { Women } \\
(n=130)\end{array}$ & $\mathbf{P}$ \\
\hline Age (years) & $\begin{array}{c}\text { Median } \\
\text { (Q1-Q3; min-max) }\end{array}$ & $\begin{array}{c}40 \\
(27-55 ; 18-87)\end{array}$ & $\begin{array}{c}48.5 \\
(26-63 ; 18-87)\end{array}$ & $\begin{array}{c}39.4 \\
(25-51 ; 18-83)\end{array}$ & $<0.001^{*}$ \\
\hline Leukocytes in urine & $\begin{array}{c}\text { Median } \\
\text { (Q1-Q3; min-max) }\end{array}$ & $\begin{array}{c}10 \\
(4-30 ; 0-40)\end{array}$ & $\begin{array}{c}8 \\
(4-30 ; 1-40)\end{array}$ & $\begin{array}{c}12 \\
(3.5-32.5 ; 0-40)\end{array}$ & $0.015^{*}$ \\
\hline Erythrocytes in urine & $\begin{array}{c}\text { Median } \\
\text { (Q1-Q3; min-max) }\end{array}$ & $\begin{array}{c}4 \\
(1-7 ; 0-40)\end{array}$ & $\begin{array}{c}4.5 \\
(2-10 ; 0-40)\end{array}$ & $\begin{array}{c}3 \\
(0-7 ; 0-40)\end{array}$ & $0.007^{*}$ \\
\hline
\end{tabular}

"Mann-Whitney U test

Table 2. Leukocyturia in relation to the detected pathogen

\begin{tabular}{|c|c|c|c|c|c|}
\hline & & $\begin{array}{c}\text { All } \\
(n=220)\end{array}$ & $\begin{array}{c}\text { U. urealyticum } \\
\text { Positive }(\mathrm{n}=186)\end{array}$ & $\begin{array}{l}\text { C. trachomatis } \\
\text { Positive }(n=34)\end{array}$ & $\mathbf{P}^{*}$ \\
\hline Leukocytes in urine & $\begin{array}{c}\text { Median } \\
\text { (Q1-Q3; min-max) }\end{array}$ & $\begin{array}{c}10 \\
(4-30 ; 0-40)\end{array}$ & $\begin{array}{c}14 \\
(4.8-30 ; 0-40)\end{array}$ & $\begin{array}{c}3 \\
(2-6 ; 1-40)\end{array}$ & $<0.001^{*}$ \\
\hline Erythrocytes in urine & $\begin{array}{c}\text { Median } \\
\text { (Q1-Q3; min-max) }\end{array}$ & $\begin{array}{c}4 \\
(1-7 ; 0-40)\end{array}$ & $\begin{array}{c}4 \\
(0-8 ; 0-40)\end{array}$ & $\begin{array}{c}3 \\
(2-5 ; 1-40)\end{array}$ & $0.901^{*}$ \\
\hline
\end{tabular}

"Mann-Whitney U test

Table 3. Chronic symptoms in male and female patients with uncomplicated urinary tract infections

\begin{tabular}{|c|c|c|c|c|}
\hline \multirow[b]{2}{*}{ Symptoms } & \multicolumn{3}{|c|}{ n (\%) } & \multirow[b]{2}{*}{$\mathbf{p}^{*}$} \\
\hline & All & Men & Women & \\
\hline Discomfort when urinating & $44(20.7)$ & $38(43.7)$ & $6(4.8)$ & \multirow{5}{*}{$<0.001$} \\
\hline Discomfort when urinating, frequency/nocturia & $34(16.0)$ & $6(6.9)$ & $28(22.2)$ & \\
\hline Discomfort when urinating, incomplete voiding & $44(20.7)$ & $5(5.7)$ & $39(31.0)$ & \\
\hline Discomfort when urinating, frequency, dysuria & $23(10.8)$ & $7(8)$ & $16(12.7)$ & \\
\hline Other & $68(31.9)$ & $31(35.6)$ & $37(29.4)$ & \\
\hline
\end{tabular}

" $\chi 2$ test

problems in the form of discomfort during urinating and frequent urinating with nocturia (two symptoms) was three times higher in women than in men. The proportion of problems in the form of discomfort during urinating and a feeling of incomplete voiding of the bladder (two symptoms) was 5.4 times higher in women than in men. The proportion of three symptoms was almost two times higher in women than in men.

After isolating the agent, therapy was included for both partners. Three months after initial therapy, 22 patients (10\%) needed repeated therapy, while 198 (90\%) patients had normal results and they did not have any symptoms. At the final check-up, after six months three patients again had Ureaplasma urealyticum. Other patients had normal microbiological test results, urine sediment was normal, without leukocyturia, and patients did not complain about any of the previously mentioned symptoms.

\section{Discussion}

In persistent or recurrent UTI in adults it is important to distinguish complicated from uncomplicated forms. Complicated UTI implies infections of urinary tract which is anatomically or functionally altered (urodynamics or voiding are abnormal). Uncomplicated UTI occur mainly in otherwise healthy patients with structurally normal urinary tract and intact voiding mechanisms [11]. Our research involved patients with uncomplicated UTI. Due to the shortness of female urethra, women are more prone to urinary tract infections as well as spreading of infection from the genital tract. Approximately $50-60 \%$ of women report at least one UTI in their lifetime, and one in three will have at least one symptomatic UTI necessitating antibiotic treatment by age 24 [12]. As in other studies, in our study, women were also more presented with chronic urinary symptoms. Also, in women, leukocyturia was more pronounced than in men.
While analysing leukocyturia, we found that the number of leukocytes was significantly higher in the group of patients with $U$. urealyticum than in the group with C. trachomatis.

A lot is already known about mycoplasma-associated genital tract infections, however there is still a lack of clarity on the influence and pathogenic potential of mycoplasma and ureaplasma species in the context of urinary tract infections and other urogynaecological symptoms such as irritable bladder and interstitial cystitis/bladder pain syndrome [3].

The role of normal vaginal flora in the defence against genital colonization with potentially pathogenic adhering $E$. coli has been demonstrated in several studies [13]. In women, disturbed vaginal microflora in fact enables colonization of uropathogens which are part of normal intestinal flora and this can lead to recurring bacterial UTI. However, even after treated bacterial infections, some symptoms of UTI and leukocyturia often persist. In such situations atypical uropathogens should be taken into consideration.

'Symptomatic abacteriuria', i.e. bacterial infection with low counts of uropathogens may present also as the so-called urethral syndrome and several pathogens are implicated such as Chlamydia, Mycoplasma, Trichomonas, Gonococci, Candida or Mycobacteria [11].

Stamm, et al. associated the urethral syndrome, that is dysuria and/or frequency without significant bacteriuria with Chlamydia trachomatis infection [14] while Matthews' found the same pathogen as cause of sterile pyuria [15].

In our research the frequency of $C$. trachomatis infection in symptomatic women was lower in relation to the proportion of $U$. urealyticum infection. Among 130 women, C. trachomatis was identified in 18 patients (14\%) while $112(86 \%)$ had $U$. urealyticum. Also, in men, 

infections

C. trachomatis was less frequent. As opposed to Matthews research, we have not proved leukocyturia in patients with chlamydia.

Our findings are in agreement with previous studies (Pott's, Baka, Schlicht) proving the fact that although often overlooked or improperly treated, U. urealyticum and M. hominis infections may account for a large proportion of unexplained chronic voiding symptoms [16-18]. In our research $U$. Urealyticum was substantially more prevalent in both genders. Of 220 patients, $186(85 \%)$ had $U$. urealyticum while $C$. trachomatis was present in 34 patients (15\%).

In our research, women had a larger number of symptoms in relation to men. In women, discomfort during urination and frequent urination occurred three times more than in men, while combined discomfort during urinating and the feeling of insufficient voiding of bladder occurred five times more in women than in men $(\mathrm{p}<0,001)$. In male patients the dominant symptom was discomfort during urination $(\mathrm{p}<0,001)$.

Our patients were given tetracycline (doxycycline twice daily) 14 days for M. hominis and $U$. urealyticum infection and 10 days for $C$. trachomatis infection. On the first check up after three months, repeated therapy was given to the total of 22 patients (10\%). After six months, three patients were again positive to U. urealyticum. Other patients had normal microbiological test results, urine sediment was normal, and patients felt no symptoms.

A question is raised when to test the patients after completed therapy and for how long to follow them. Do we often get a falsely negative result if we take samples too early? It often happens that both partners are not treated.

According to our experience, we think that sample testing should be carried out after three months from the therapy, while earlier testing should be carried out only in patients who experience repeated symptoms. We underline the need to treat both partners.

\section{Conclusion}

In patients with frequent and repeated uroinfections it is advisable to assess the presence of $U$. urealyticum, M. hominis and C. trachomatis. Persisting leukocyturia is more often found in U. urealyticum infection, while normal urine sediment in symptomatic patients may refer to $C$. trachomatis infection.

\section{Acknowledgements}

Hana Đogaš, School of Medicine, University of Split, Split, Croatia, responsible for transfering data from working draft tables to Excel tables and literature searching. Tanja Barišić, School of Medicine, University of Split, Split,Croatia, responsible for transfering data from working draft tables to Excel tables.

\section{References}

1. McCormack WM, Rein MF (2000) Urethritis. In: Mandell GL, Bennett J E, Dolin R (Eds). Principles and practice of infectious diseases (5th Edn) New York: Churchill Livingstone pp.1208-1218.

2. Kilic D, Basar MM, Kaygusuz S, Yilmaz E, Basar H, et al. (2004) Prevalence and treatment of Chlamydia trachomatis, Ureaplasma urealyticum, and Mycoplasma hominis in patients with non-gonococcal urethritis. Jpn J Infect Dis 57: 17-20.

3. Combaz-Söhnchen N, Kuhn A (2017) A systematic review of mycoplasma and ureaplasma in urogynaecology. Geburtshilfe Frauenheilkd 77: 1299-1303.

4. Hatch TP (1999) Developmental biology. In: Stephens RS (Ed.) Chlamydia: Intracellular Biology, Pathogenesis, and Immunity. ASM Press, Washington DC pp. 29-67.

5. Bébéar C, de Barbeyrac B (2009) Genital chlamydia trachomatis infections. Clin Microbiol Infect 15: 4-10.

6. Glen P, Prashar A, Hawary A (2016) Sterile pyuria: a practical management guide. Br J Gen Pract 66: e225-e227. [Crossref]

7. Pappas PG (1991) Laboratory in the diagnosis and management of urinary tract infections. Med Clin North Am 75: 313-325. [Crossref]

8. Nassar FA, Abu-Elamreen FH, Shubair ME, Sharif FA (2008) Detection of Chlamydia trachomatis and Mycoplasma hominis, genitalium and Ureaplasma urealyticum by polymerase chain reaction in patients with sterile pyuria. Adv Med Sci 53: 80-86.

9. Ronald RA (2002) Urinary tract infections in adults. In: Tan JS, editor. Experts guide to the management of common infectious diseases, Philadelphia: American College of Physicians pp. 229-250.

10. Tomas ME, Getman D, Donskey CJ, Hecker MT (2015) Overdiagnosis of urinary tract infection and underdiagnosis of sexually transmitted infection in adult women presenting to an emergency department. J Clin Microbiol 53: 2686-2692.

11. Franz M, Hörl WH (1999) Common errors in diagnosis and management of urinary tract infection. I: Pathophysiology and diagnostic techniques. Nephrol Dial Transplant 14: 2746-2753.

12. Rahn DD (2008) Urinary tract infections: contemporary management. Urol Nurs 28 333-341.

13. Gupta K, Stapleton AE, Hooton TM, Roberts PL, Fennell CL, et al. (1998) Inverse association of $\mathrm{H}_{2} \mathrm{O}_{2}$-producing lactobacilli and vaginal Escherichia coli colonization in women with recurrent urinary tract infections. J Infect Dis 178: 446-450.

14. Stamm WE, Wagner KF, Amsel R, Alexander ER, Turck M, et al. (1980) Causes of the acute urethral syndrome in women. $N$ Engl J Med 303: 409-415. [Crossref]

15. Matthews RS, Bonigal SD, Wise R (1990) Sterile pyuria and Chlamydia trachomatis. Lancet 336: 385. [Crossref]

16. Potts JM, Ward AM, Rackley RR (2000) Association of chronic urinary symptoms in women and Ureaplasma urealyticum. Urology 55: 486-489.

17. Baka S, Kouskouni E, Antonopoulou S, Sioutis D, Papakonstantinou M, et al. (2009) Prevalence of Ureaplasma urealyticum and Mycoplasma hominis in women with chronic urinary symptoms. Urology 74: 62-66.

18. Schlicht MJ, Lovrich SD, Sartin JS, Karpinsky P, Callister SM, et al. (2004) High prevalence of genital mycoplasmas among sexually active young adults with urethritis or cervicitis symptoms in La Crosse, Wisconsin. J Clin Microbiol 42: 4636-4640. [Crossref]

Copyright: (C2019 Vlasic-Matas J. This is an open-access article distributed under the terms of the Creative Commons Attribution License, which permits unrestricted use, distribution, and reproduction in any medium, provided the original author and source are credited. 